\title{
Kauffman Bracket of Plane Curves
}

\author{
S. Chmutov ${ }^{1, \star}$, V. Goryunov ${ }^{2, \star \star}$ \\ ${ }^{1}$ Program Systems Institute, Pereslavl-Zalessky, 152140, Russia \\ E-mail: chmutov@math.botik.yaroslavl.su \\ 2 Department of Mathematics, The University of Liverpool, Liverpool, L69 3BX, UK \\ E-mail: goryunov@liv.ac.uk
}

Received: 15 November 1995/Accepted: 26 January 1996

\begin{abstract}
We lower the Kauffman bracket for links in a solid torus (see [16]) to generic plane fronts. It turns out that the bracket can be entirely defined in terms of a front itself without using the Legendrian lifting. We show that all the coefficients of the lowered bracket are in fact Vassilev type invariants of Arnold's $J^{+}$-theory $[3,4]$. We calculate their weight systems. As a corollary we obtain that the first coefficient is essentially the quantum deformation of the Bennequin invariant introduced recently by M. Polyak [19].
\end{abstract}

There exists a straightforward way to get an invariant of an immersed cooriented hypersurface $C$ in a smooth manifold $N$. We lift $C$ to the manifold $M$ of cooriented contact elements of $N$. This gives us an embedded submanifold $L_{C}$. Now we take the value of a known invariant of embeddings on $L_{C} \hookrightarrow M$ as the invariant of our initial immersion $C \rightarrow N$.

The manifold $M$ of cooriented contact elements is the spherisation of the cotangent bundle of $N: M=S T^{*} N$. It has a natural contact structure. Our lifting $L_{C}$ is a Legendrian submanifold with respect to this structure. The hypersurface $C$ is called the front of $L_{C}$. The above procedure defines an invariant not only on immersed $C \rightarrow N$ but also on submanifolds with some "admissible" singularities which may appear as singularities of fronts of smooth Legendrian submanifolds generically embedded into $M$.

The simplest situation is $N=\mathbf{R}^{2}$. The "admissible" singularities in this case are cusps. Thus we can induce an invariant on collections of closed oriented and cooriented plane curves which may have only double points and cusps as singularities. The manifold $M$ of contact elements of the plane is the solid torus $M=\mathbf{R}^{2} \times S^{1}$. So the lifted submanifolds are Legendrian links in it. This general approach was used in [12] to define an invariant of an immersed plane curve. There a Kontsevich type integral [11] was taken as a known invariant of knots in a solid torus. In a

\footnotetext{
^Partially supported by the Fellowship "Emma e Giovanni Sansone," the International Science Foundation and the Russian Foundation for Fundamental Research.

$\star \star$ Supported by an RDF grant of The University of Liverpool.
} 
similar way, a polynomial invariant of knots in a solid torus defined in [1] was lowered to plane curves in [2].

In this paper we take the Kauffman bracket for links in a solid torus (see [16]) as a known invariant to be induced on plane fronts. It turns out that it can be entirely defined in terms of front $C$ itself without using the Legendrian lifting. The Kauffman bracket is a polynomial in two variables $A$ and $h$, Laurent in $A$ and ordinary in $h$. We show that, after the substitution $A=e^{t}$ and Taylor expansion in a power series in $t$, the coefficient at $t^{n}$ is an invariant of Arnold's $J^{+}$-theory $[3,4]$ of order at most $n$ in the Vassiliev sense. These coefficients are polynomials in $h$. We calculate the corresponding symbols (weight systems). As a corollary we obtain that the first coefficient is essentialy the quantum deformation of the Bennequin invariant introduced recently by M. Polyak [19]. In the last section we lower other polynomial invariants of links to plane fronts and formulate a series of conjectures about them.

For an application of the same general idea to induce order 1 invariants in a higher-dimensional situation see [13].

\section{Definitions and Known Results}

In this section we recall some basic facts about our curves, corresponding Legendrian links and their invariants. See [3, 4] for more details.

1.1. Legendrian links and their fronts. A contact element at a point of a plane is a line in the tangent plane. Its coorientation is a choice of one of two half-planes into which it divides the tangent plane. The manifold $M=S T^{*} \mathbf{R}^{2}$ of all cooriented contact elements of the plane is diffeomorphic to the solid torus $\mathbf{R}^{2} \times S^{1}$, since the coorienting normal vector is defined by its angle $\varphi$. Manifold $M$ has a natural contact structure defined as zeros of the form $(\cos \varphi) d x+(\sin \varphi) d y$, where $(x, y)$ are coordinates on $\mathbf{R}^{2}$. A Legendrian link $L$ in $M$ is an embedding of a number of oriented circles into $M$ tangent to the contact planes at each of its points. A Legendrian link has a natural framing by transversals to the contact planes. The canonical projection of $L$ to $\mathbf{R}^{2}$ gives a collection of plane curves. We call it the front of $L$. It has an orientation (coming from $L$ ) and a coorientation (the coordinate $\varphi$ forgotten by the projection defines not only the line tangent to the front, but the side of this line as well). A generic front may have only transverse double points and cusps as singularities. We call such a front a normal front. Since a front is cooriented the number of cusps on each component of a normal front is even.

Any cooriented plane curve $C$ lifts to a Legendrian curve $L_{C} \in M$ by taking the cooriented tangent direction as a contact element at each point of $C$. The lifting of a collection of curves with normal front singularities is a link (Fig. 1).

1.2. Index, Maslov index and perestroikas. To each component $C_{j}$ of a normal front $C=\cup C_{j}$ we assign two integers, index $1 \operatorname{ind}\left(C_{j}\right)$ and Maslov index $\mu\left(C_{j}\right)$. ind $\left(C_{j}\right)$ is the number of full rotations made by the coorienting vector as it moves along $C_{j}, \mu\left(C_{j}\right)$ is the difference between the numbers of positive and negative cusps of $C_{j}$. A cusp is called positive if the 1-form which coorients the curve at the

\footnotetext{
${ }^{1}$ Other names are winding number, rotation number, Whitney index.
} 

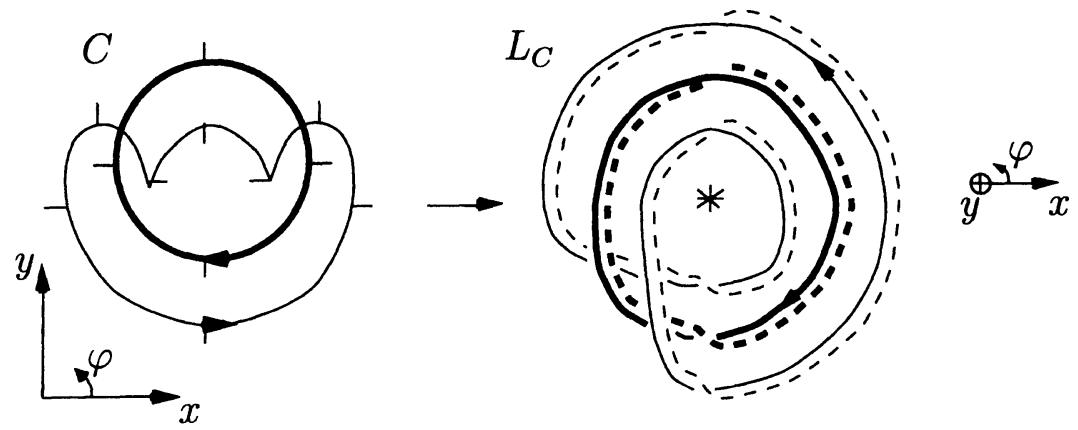

Fig. 1. Legendrian lifting

Left picture: A normal front $C$ with two components; $(x, y)$ are coordinates on $\mathbf{R}^{2} ; C$ lies in the halfplane $x>0$.

Right picture: Framed Legendrian link $L_{C}$ is drawn as a diagram of the projection to the punctured plane with polar coordinates $(x, \varphi)$; the $y$-axis is perpendicular to the plane and directed from the reader.

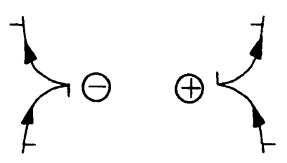

Fig. 2. Negative and positive cusps

cusp point is positive on the neighbouring orienting vectors and negative otherwise (Fig. 2).

The number $\mu\left(C_{j}\right)$ is always even. Reversing of the orientation of $C_{j}$ changes the signs of both ind $\left(C_{j}\right)$ and $\mu\left(C_{j}\right)$. Reversing of the coorientation of $C_{j}$ changes only the sign of $\mu\left(C_{j}\right)$.

There are four types of generic degenerations of a normal front. We show them in Fig. 3 in perestroikas in generic 1-parameter families.

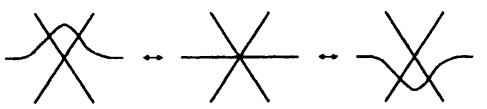

triple point perestroika

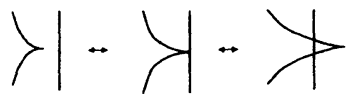

cusp crossing perestroika

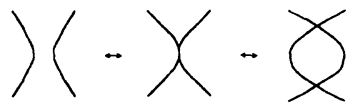

self-tangency perestroika

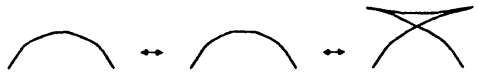

cusp birth-death perestroika

Fig. 3. Perestroikas

Theorem [14] (see also $[3,4])$. The collection of pairs $\left(\operatorname{ind}\left(C_{j}\right), \mu\left(C_{j}\right)\right.$ ) is a complete invariant of a normal front $C=\cup C_{j}$ under plane isotopies and the four types of perestroikas of Fig. 3. 
For each pair of integers $r \geqq 1$ and $s \geqq 0$ let $K_{r, s}$ be the curve of Fig. 4 . $\operatorname{ind}\left(K_{r, s}\right)=r-1 ; \mu\left(K_{r, s}\right)=2 s$.

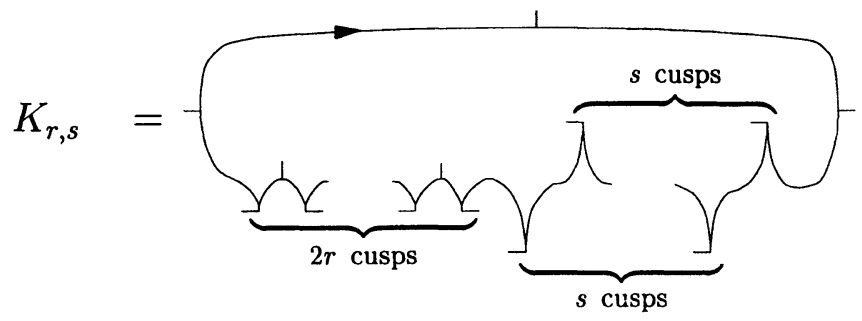

Fig. 4. Canonical curves ${ }^{2}$

The theorem says that each component $C_{j}$ of a normal front can be transformed to one of the curves $K_{r, s}$ (possibly with changed orientation or coorientation or both) by a sequence of the perestroikas and isotopies of the plane. Figure 5 provides an example.

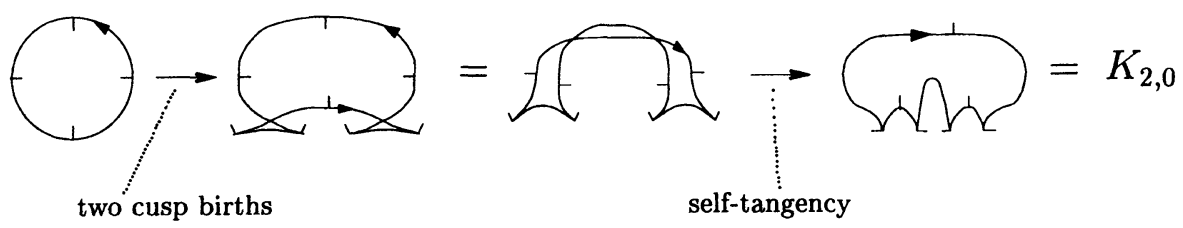

Fig. 5. Transformation of the circle to $K_{2,0}$

1.3. $\mathrm{J}^{+}$-type invariants. It is convenient to subdivide self-tangency perestroikas into the following four types according to the orientations and coorientations. A selftangency is called dangerous if both the tangent branches are cooriented by the same half-plane and safe otherwise. A self-tangency is called direct if both tangent branches are oriented by the same tangent vector and inverse otherwise.

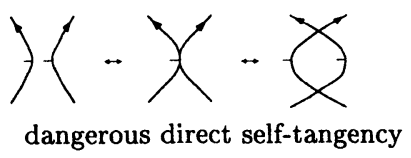

$j(\cdots)$

safe direct self-tangency

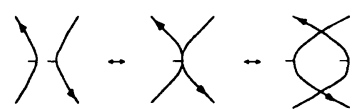

dangerous inverse self-tangency

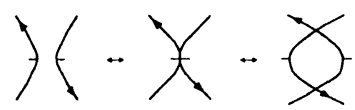

safe inverse self-tangency

Fig. 6. Four types of self-tangencies

Note that, if two tangent branches belong to the same component of a front, the property of the tangency point to be direct or inverse (resp. dangerous or safe) does not depend on the orientation (resp. coorientation) of the component.

\footnotetext{
${ }^{2}$ Our choice of canonical curves slightly differs from Arnold's one [3].
} 
It is easy to see that the topological type of a Legendrian link $L_{C}$ in the solid torus $M$ does not change under all the perestroikas except dangerous self-tangencies. A dangerous self-tangency perestroika corresponds to an interchanging of overcrossing and undercrossing in a link diagram like that in Fig. 1. But not all interchangings can be done in the class of Legendrian links, and so in the class of corresponding fronts.

We will say that two fronts are $J^{+}$-equivalent if one can be transformed to another without dangerous self-tangencies. Figure 5 shows a $J^{+}$-equivalence of the circle to $K_{2,0}$. Similarly one can show that the circle with the opposite coorientation is also $J^{+}$-equivalent to $K_{2,0}$. Another example is $J^{+}$-equivalence of figure-eight curves with different choices of orientation and coorientation (Fig. 7).

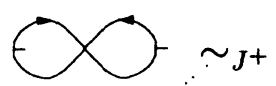

cusp births

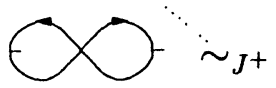

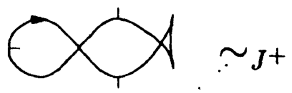

safe self-tangencies

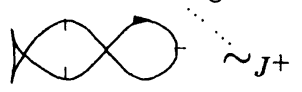<smiles>C1CCCC1</smiles>

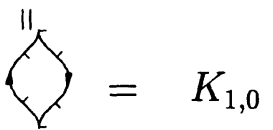

Fig. 7. $J^{+}$-equivalence of figure-eight curves

By a $J^{+}$-type invariant we mean an invariant of normal fronts which does not change under all the perestroikas except dangerous self-tangencies. The first example of such an invariant was an invariant introduced by V.I. Arnold in [3, 4] and named $J^{+}$. This is an invariant of a one component front defined by its values on the canonical curves:

$$
J^{+}\left(K_{r, s}\right)=-s \quad \text { (for any choice of the orientation and coorientation) }
$$

and by its behavior under the dangerous self-tangency perestroikas:

$$
J^{+}(\Varangle)-J^{+}\left(-()=2 ; \quad J^{+}\left(y-()-J^{+}(ל)=2 .\right.\right.
$$

According to Theorem 1.2 this data is sufficient for calculating $J^{+}$on any normal front. Here is an example.

$$
\begin{aligned}
& J^{+}(\leadsto)=J^{+}(\hat{\theta})=J^{+}(\hat{q})-2 \\
& \text { two cusp crossings dangerous self-tangency }
\end{aligned}
$$

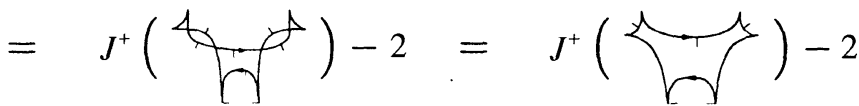

$$
\begin{aligned}
& \text { two cusp births } \\
& \text { two safe self-tangencies } \\
& =J^{+}\left(K_{3,0}\right)-2=-2 \text {. }
\end{aligned}
$$

There are several combinatorial formulas for calculating the values of $J^{+}$on curves without cusps (see a review in [8]) and Polyak's formula [18] for curves with cusps. 
In the Vassiliev sense $J^{+}$is an invariant of order 1.

Remark. Reversing orientations of both the local branches in the two dangerous self-tangency perestroikas of Fig. 6, one obtains two more dangerous perestroikas which look different from those above. But their behaviour in all our constructions is absolutely identical to the behaviour of the corresponding "twins". So we spell all the formulas involving dangerous self-tangencies only for the two perestroikas of Fig. 6.

1.4. The Bennequin invariant and its quantization. For a Legendrian knot $K$ in a contact $\mathbf{R}^{3}$ Bennequin [6] defined a self-linking number $\beta$ as the linking number of $K$ with a small shift of $K$ in a direction everywhere transversal to the contact planes. This definition was generalized to a non simply-connected case of Legendrian knots in the solid torus $S T^{*} \mathbf{R}^{2}$, with its standard contact structure, by S. Tabachnikov [20].

As the usual linking number [17] the Bennequin-Tabachnikov invariant can be read from a diagram of a knot and its framing like that in Fig. 1. For example, for the bold component of the link of Fig. $1 \beta=1$ (we have two positive crossings of the projections of the knot and its framing), for the thin component $\beta=3$ (we have six possitive crossings there).

Arnold proved [3] that $\beta=1-J^{+}$. So any combinatorial formula for $\beta$ gives a formula for $J^{+}$and vice versa. Several such formulas are in [20] (see also [9]).

M. Polyak [19] invented the following state sum formula for the BennequinTabachnikov invariant $\beta$. To each crossing $p$ of a one component normal front $C$ we attach the sign $\sigma(p)=+1$, if the pairs (orienting vector, coorienting vector) for the two intersecting branches give the same orientation of the plane, and $\sigma(p)=-1$ otherwise. According to this sign we split $C$ at $p$ respecting the orientation and coorientation (Fig. 8).

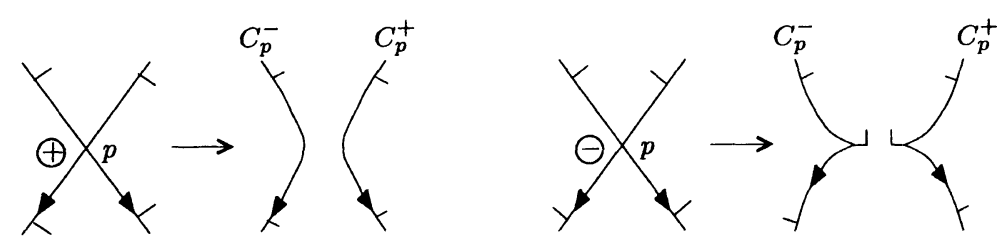

Fig. 8. Splittings of a front $C$ at a crossing saving the orientation and coorientation

In fact this is a unique natural splitting which gives two component curves with two branches near $d$ belonging to different components. Denote by $C_{p}^{-}\left(\operatorname{resp} . C_{p}^{+}\right)$ the component that contains the left (resp. right) branch assuming both branches oriented downwards (see Fig. 8).

Theorem [19]. Let $C$ be a one component normal front. Denote by $S$ the state sum

$$
S=\sum_{p}\left(\operatorname{ind}\left(C_{p}^{+}\right)-\operatorname{ind}\left(C_{p}^{-}\right)-\sigma(p)\right)
$$

over the set of all double points of $C$. Then

$$
\beta\left(L_{C}\right)=S-(\operatorname{ind}(C)-1) n^{+}+(\operatorname{ind}(C)+1) n^{-}+\operatorname{ind}^{2}(C),
$$


where $n^{+}$(resp. $n^{-}$) is half the number of cusps of $C$ whose neighbourhoods give a positive (resp. negative) contribution to the index of $C$.

This formula admits a quantum deformation [19]. Let $q$ be a formal quantum parameter and

$$
[n]_{q}=\frac{q^{n}-q^{-n}}{q-q^{-1}} \in \mathbf{Z}\left[q, q^{-1}\right]
$$

the corresponding quantum integer.

Theorem [19]. Let $S_{q}=\sum\left[\left(\operatorname{ind}\left(C_{p}^{+}\right)-\operatorname{ind}\left(C_{p}^{-}\right)-\sigma(p)\right]_{q}\right.$ be a quantum state sum. Then

$$
\beta_{q}\left(L_{C}\right)=S_{q}-[\operatorname{ind}(C)-1]_{q} n^{+}+[\operatorname{ind}(C)+1]_{q} n^{-}+[\operatorname{ind}(C)]_{q} \operatorname{ind}(C)
$$

is a $J^{+}$-type invariant of a one component normal front $C$ such that $\beta_{1}\left(L_{C}\right)=$ $\beta\left(L_{C}\right)$.

Remark. The definition of $\beta_{q}$ is easily seen to be independent from orientation and coorientation of a normal front.

Taking our canonical curves $K_{r, s}$ with the orientations as in Fig. 4 we get $n^{+}=$ $(2 r+s) / 2, n^{-}=s / 2$ and ind $(C)=r-1$. So

$$
\beta_{q}\left(L_{K_{r, s}}\right)=-\left(r+\frac{s}{2}\right)[r-2]_{q}+\frac{s}{2}[r]_{q}+(r-1)[r-1]_{q} .
$$

Let us describe the behavior of the quantum Bennequin invariant under dangerous self-tangencies. First of all we define an index $i_{d}$ of a self-tangency point $d$ which appears during such a perestroika of a normal front. To do this we split the selftangency point respecting the orientation and coorientation as shown in Fig. 9.

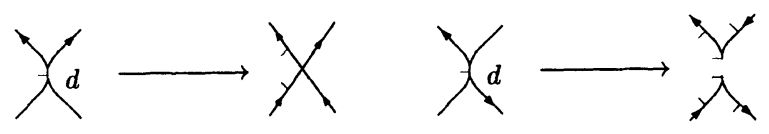

Fig. 9. Splittings of dangerous self-tangencies saving the orientation and coorientation

We obtain two curves. Let $i^{\prime}$ and $i^{\prime \prime}$ be their indices. We set $i_{d}=\left|i^{\prime}-i^{\prime \prime}\right|$. The jumps of $\beta_{q}$ under dangerous self-tangencies of the index $i_{d}$ are:

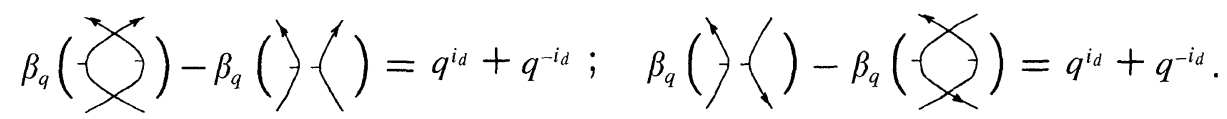

These formulas show that $\beta_{q}$ is an invariant of order 1 in the Vassiliev sense.

\section{Kauffman Bracket}

In this section we define the Kauffman bracket and prove its uniqueness. The bracket does not depend on the orientations of curves of a collection.

For a framed link in a solid torus the Kauffman bracket was defined in [16]. Its values belong to $\mathbf{Z}\left[A^{ \pm 1}, h\right]$. Using the Legendrian lifting we can define $\langle C\rangle=\left\langle L_{C}\right\rangle$. This is a $J^{+}$-type invariant of a normal front $C$. We call it the Kauffman bracket of $C$. 


\subsection{Main result}

Theorem 1. There exists a unique $J^{+}$-type invariant $\langle C\rangle \in \mathbf{Z}\left[A^{ \pm 1}, h\right]$ of a normal front $C$ satisfying the following properties:

1) $\langle\searrow\rangle=A^{-1}\langle\rangle\langle\rangle-A^{-2}\left\langle\begin{array}{l}\searrow \\ \lambda\end{array}\right.$

2) $\langle\infty\rangle=-A^{3}$;

3) $\langle\bigcirc\rangle=-A^{3} h$;

4) $\left\langle C_{1} \cdot C_{2}\right\rangle=-\left(A^{2}+A^{-2}\right)\left\langle C_{1}\right\rangle \cdot\left\langle C_{2}\right\rangle$, for $C_{1} \neq \emptyset, C_{2} \neq \emptyset$.

Here $C_{1} \cdot C_{2}$ is a collection of two fronts $C_{1}$ and $C_{2}$ which lie in different halfplanes with respect to a certain line in $\mathbf{R}^{2}$.

Remarks. 1. After the Legendrian lifting (see Fig. 1) the fragments of links corresponding to the fronts of property 1$)$ have the following diagrams in the $(x, \varphi)$-plane:

$$
\langle\rangle\left\langle=A^{-1}\langle\rangle\right\rangle-A^{-2}\langle\circlearrowright\rangle \text {. }
$$

So property 1) is just the usual skein relation for the Kauffman bracket. All other properties also correspond to the usual properties of the Kauffman bracket in the solid torus (see [16]). So the existence of such a bracket of normal fronts follows directly from [16].

2. For calculation of the Kauffman bracket we will use the fact that the curve $K_{1,0}$ can be moved through other curves of a front. For example

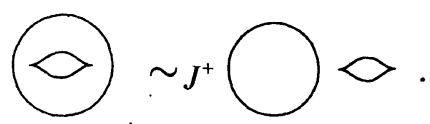

two cusp crossings

So, if one of the components of our front is the $K_{1,0}$ with nothing inside, we can transfer it far away from everything else and apply property 4) of Theorem 1:

$$
\langle\bigcirc\rangle=-A^{6} h\left(A^{2}+A^{-2}\right)=-\left(A^{4}+A^{8}\right) h \text {. }
$$

3. One more useful fact is that two circles with opposite coorientations are $J^{+}$ equivalent (see Sect. 1.3 and Fig. 5). So their brackets are equal.

Proposition 1. For any $J^{+}$-type invariant satisfying properties 1)-4) of Theorem 1 the following equalities hold:

$$
\begin{aligned}
& \langle\searrow\rangle=A\langle\circlearrowright\rangle-A^{2}\langle\stackrel{\searrow}{\searrow}\rangle \text {; } \\
& \langle\rangle\langle\rangle-\langle\rangle\rangle=\left(A-A^{-1}\right)\left(\langle\searrow\rangle-\left\langle\begin{array}{l}
\searrow\rangle \\
\lambda\rangle
\end{array}\right) .\right.
\end{aligned}
$$


Proof of Proposition 1.

$$
\begin{aligned}
& \left.\langle\rangle^{\rangle}\right\rangle=A^{-1}\left\langle\zeta_{\gamma}\right\rangle-A^{-2}\left\langle\zeta_{\gamma}^{\gamma}\right\rangle
\end{aligned}
$$

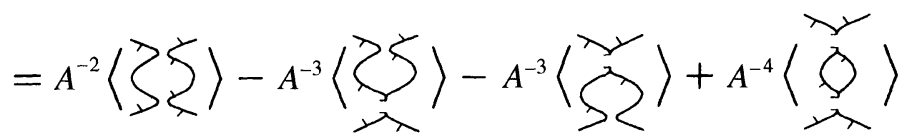

$$
\begin{aligned}
& =A^{-1}\left(A^{-1}\langle\rangle\langle\rangle-A^{-2}\left\langle\begin{array}{l}
\searrow \\
\nearrow
\end{array}\right)\right. \\
& +\left(-A^{-3}\left\langle\begin{array}{l}
\searrow \\
\nearrow
\end{array}\right\rangle+A^{-4}\left(-A^{3}\right)\left(-A^{2}-A^{-2}\right)\langle\underset{\searrow}{\searrow}\rangle\right) \\
& =A^{-1}\langle\searrow\rangle+\left(-A^{-3}+A+A^{-3}\right)\left\langle\begin{array}{l}
\searrow \\
\searrow
\end{array}\right\rangle \text {. }
\end{aligned}
$$

This implies the first equality of the proposition. The second one follows from it and property 1 ) of Theorem 1 .

2.2. Useful lemmas. In lemmas below we prove some relations for the Kauffman bracket which follow from properties 1)-4). We will use these relations in Sect. 2.3 to prove the uniqueness of the Kauffman bracket.

The relations hold for both possible coorientations of the fragment involved. Therefore we do not indicate its coorientation. The coorientation of the extra circular component also does not matter due to Remark 3 above.

Lemma 1. $\langle\curvearrowleft\rangle=A\langle\underline{O}\rangle-A^{2}\langle\boldsymbol{\Lambda}\rangle$.

Proof of Lemma 1. $\langle\Lambda\rangle=\langle\Omega\rangle=A^{-1}\langle\underline{O}\rangle-A^{-2}\langle\curvearrowleft\rangle$.

safe self-tangency and cusp death

Lemma $2 . \hat{\gamma}\rangle=\langle\gamma\rangle=\left(-A^{3}\right)\langle-\rangle$.

Proof of Lemma 2.

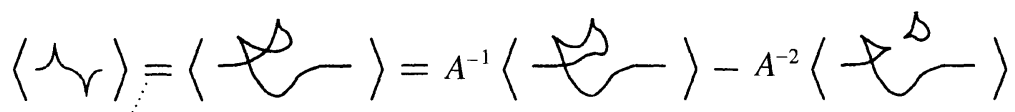

cusp crossing

$$
=\left(A^{-1}-A^{-2}\left(A^{5}+A\right)\right)\langle-\rangle=\left(-A^{3}\right)\langle-\rangle .
$$

cusp death and properties 4),2)

The proof of the second equality is similar. 
Lemma 3. $\langle\mathfrak{M}\rangle=A^{-1}\langle\stackrel{O}{O}\rangle-A^{4}\langle-\rangle$.

Proof of Lemma 3.

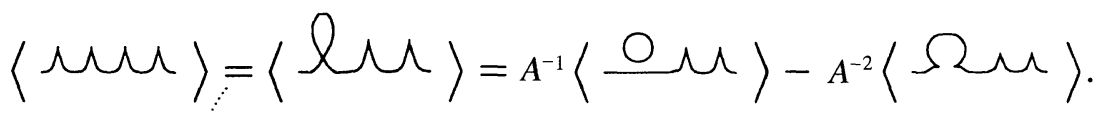

$$
\begin{aligned}
& \text { safe self-tangency and cusp death }
\end{aligned}
$$

The last term is equal to $A^{-2}\left(-A^{3}\right)^{2}\langle-\rangle=A^{4}\langle-\rangle$ by Lemma 2 .

Lemma 4. $\left\langle\frac{}{\bigcirc}\right\rangle=\left(A^{-1}-A^{3}\right)\langle\mathcal{\Lambda}\rangle-A^{2}\langle\underline{\bigcirc}\rangle$.

Proof of Lemma 4.

$$
\begin{aligned}
\langle\bar{O}\rangle & =A\langle\curlyvee\rangle+A^{-1}\langle\longrightarrow\rangle \\
& =A\langle\curlyvee\rangle+A^{-1}\langle\curlywedge\rangle
\end{aligned}
$$

cusp birth and safe self-tangency

$$
=A^{2}\left\langle\stackrel{\bigcirc}{\bigcirc}+\left(A^{-1}-A^{3}\right)\langle\mathcal{\Lambda}\rangle\right.
$$

Lemma 1

2.3. Proof of Theorem 1. To prove the uniqueness of the bracket it is enough to show that properties 1)-4) are sufficient for calculation of the bracket on any normal front. We prove this giving an algorithm for such calculation.

First of all we eliminate all double points of the front using the skein relation 1). We obtain a linear combination of brackets of fronts without double points. Each of these fronts is just a union of "ovals" which can have cusps and be nested. Using Lemma 2 we cancel pairs of neighbouring cusps with opposite directions. Then using Lemma 1 we invert the directions of pairs of cusps from inside to outside of their "oval".

After that we reduce the number of cusps on each "oval" to zero or two (Lemma 3 ). Now consider the deepest "ovals" of the nests. We transfer all those which are $K_{1,0}$-curves far away (see Remark 2 ) reducing our computation to the computation of the bracket of the remaining part. We have left only circles on the deepest level. We decrease their depth by Lemma 4 . This brings us to the beginning of this paragraph with the depth of the nests reduced by 1 . Theorem 1 is proved.

Corollary. The Kauffman bracket does not distinguish between two fronts which differ by the simultaneous change of coorientations of all the components.

Corollary follows directly from the proof of Theorem 1.

Example. The Kauffman bracket of canonical curves.

$$
\left\langle K_{r, s}\right\rangle=\left(-A^{3}\right)^{s}\left\langle K_{r, 0}\right\rangle \text { by Lemma } 2 \text {; }
$$

$$
\left.\left.\left\langle K_{1,0}\right\rangle=-A^{3} \text { by property } 2\right) ; \quad\left\langle K_{2,0}\right\rangle=-A^{3} h \text { by property } 3\right) \text {. }
$$


For $r>2,\left\langle K_{r, 0}\right\rangle$ can be computed recurrently:

$$
\begin{aligned}
& \left\langle K_{r, 0}\right\rangle=\langle\underbrace{r \quad r}_{2 r \text { cusps }}\rangle \\
& =A^{-1}\langle\underbrace{\sim r v}_{2(r-1) \text { cusps }}\rangle-A^{-4}\left\langle K_{r-2,0}\right\rangle \\
& =\left(A^{4}+1\right) h\left\langle K_{r-1,0}\right\rangle-A^{-4}\left\langle K_{r-2,0}\right\rangle .
\end{aligned}
$$

properties 4) and 3)

Setting $A=1$ we get $\left.\left\langle K_{r, 0}\right\rangle\right|_{A=1}=-T_{r-1}(h)$, where the $T_{n}(h)$ are the classical Tchebyshev polynomials in $h: T_{n}(\cos x)=\cos (n x)$. So the negative of $\left\langle K_{r, 0}\right\rangle$ can be considered as a deformation of the Tchebyshev polynomial with the parameter $A$. The number of the polynomial is the absolute value of the index of the canonical curve.

\section{Taylor Coefficients}

In this section we prove an analog of the Birman-Lin theorem [7] for the Kauffman bracket of a normal front and calculate the symbols of Taylor coefficients of the bracket as functions on marked chord diagrams.

3.1. Finite order $J^{+}$-type invariants. The extension of a knot invariant to degenerate knots with double points is basic for the Vassiliev theory. In a similar way any $J^{+}$ type invariant $f$ recursively extends to fronts with a finite number of dangerous self-tangencies:

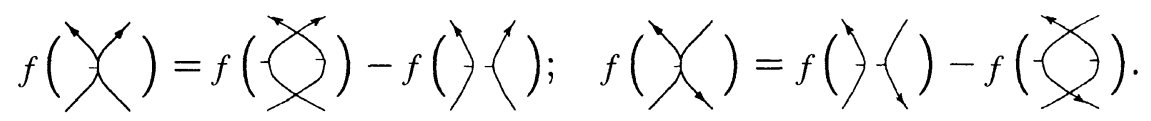

These rules are due to the natural coorientation of the strata of dangerous selftangencies from [3]. When lifted to $S T^{*} \mathbf{R}^{2}$ both rules are in fact the definition of an extended invariant of the original Vassiliev theory.

Following the above rules we get the extension of the Kauffman bracket of plane fronts. One should note that, though the Kauffman bracket of a normal front does not depend on orientations of its components, the extended bracket does depend on these orientations if a degenerate front has more than one component.

Definitions. $A J^{+}$-type invariant $f$ has order $n$ in Vassiliev sense if $n$ is the maximal number of dangerous self-tangencies of a front on which the extension of $f$ does not vanish. The symbol of such $f$ is the restriction of $f$ to the set of fronts with precisely $n$ dangerous self-tangencies.

Gromov's theorem (Sect. 1.2) means that indices and Maslov indices of the components are the only invariants of order zero. 
The difference of two invariants of order $n$ with the same symbol is an invariant of order less than $n$.

Theorem 2. Set $A=e^{t}$ in the Kauffman bracket of a plane front $C$ and expand the result in a power series in $t$. Then the coefficient at $t^{n}$ in the series $\left.\langle C\rangle\right|_{A=e^{t}}$ is a $J^{+}$-type invariant of order at most $n$ in Vassiliev sense.

Proof of Theorem 2. Let $C$ be a front with $n+1$ dangerous self-tangency points $d_{1}, \ldots, d_{n+1}$. We consider two splittings of $C$ (Fig. 10) at a point $d_{i}$ and attach to each of the splittings a sign $\varepsilon\left(d_{i}\right)$ which indicates either agreement or disagreement of the surgery with the orientations.

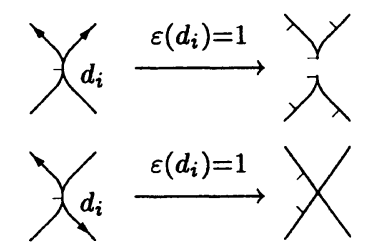

disagreement with the orientations

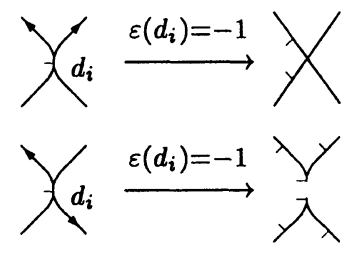

agreement with the orientations

Fig. 10. Signs of splittings of dangerous self-tangencies

Let $C_{\varepsilon_{1}, \ldots, \varepsilon_{n+1}}$ be the splitting of $C$ at all the points $d_{1}, \ldots, d_{n+1}$ with the signs $\varepsilon\left(d_{i}\right)=\varepsilon_{i}$. The second equality of Proposition 1 of Sect. 2.1 implies

$$
\langle C\rangle=\left(A-A^{-1}\right)^{n+1} \sum_{\varepsilon_{1}, \ldots, \varepsilon_{n+1}} \varepsilon_{1} \cdot \ldots \cdot \varepsilon_{n+1} \cdot\left\langle C_{\varepsilon_{1}, \ldots, \varepsilon_{n+1}}\right\rangle,
$$

where the sum is taken over all $2^{n+1}$ possible splittings of the self-tangency points. The substitution $A=e^{t}$ and Taylor expansion provide

$$
\left(A-A^{-1}\right)^{n+1}=(2 t)^{n+1}+\text { terms of higher degree . }
$$

Therefore the coefficient at $t^{n}$ in $\left.\langle C\rangle\right|_{A=e^{t}}$ is equal to zero. Theorem 2 is proved.

Remark. The proof demonstrates a bit more than the theorem claims. Namely, evaluations at $A=1$ and $A=-1$ of the $n^{\text {th }}$ derivative of the Kauffman bracket with respect to $A$ turn out to be invariants of order at most $n$ (cf. [7]). The exponential substitution is a sort of tradition introduced in [7].

3.2. Symbols of the coefficients. There are several ways to define a chord diagram of a degenerate front. Say, one can follow the approach of [12] marking chords. But the way which looks most convenient for the study of the Kauffman bracket is as follows.

Consider an oriented $l$-component front $C$ with $n$ dangerous self-tangencies. Up to an isotopy of the ambient plane we can assume that the coorienting vector at each of the self-tangency points is horizontal and directed to the left. Take $l$ disjoint circles $S_{1}^{1} \cup \cdots \cup S_{l}^{1}$ oriented counter-clockwise in a plane. Consider the front $C$ as the image of a mapping $S_{1}^{1} \cup \cdots \cup S_{l}^{1} \rightarrow \mathbf{R}^{2}$. Connect the two preimages of a direct (resp. inverse) dangerous self-tangency by a solid (resp. dashed) chord. Orient this chord from the inverse image of the right-hand branch of the self-tangency to that 
of the left-hand one. Mark an arc of a circle between two neighbouring endpoints of chords by a pair of integers $(i, \mu)$, where $i$ is the contribution of this arc to the index of $C$ and $\mu$ is its contribution to the Maslov index of $C$.

The obtained chord diagram considered up to orientation-preserving diffeomorphisms of the circles $S_{1}^{1}, \ldots, S_{l}^{1}$ is called the marked chord diagram of the front $C$ and denoted by $D_{C}$. Any abstract marked chord diagram is easily seen to be the marked chord diagram of an appropriate front.
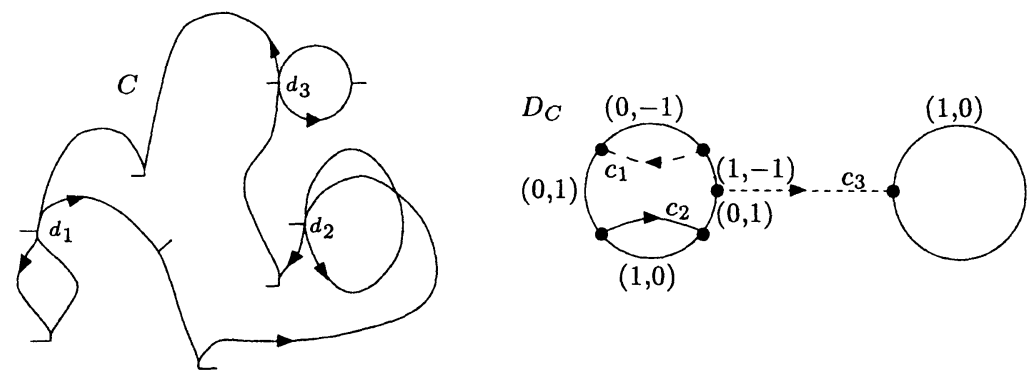

Fig. 11. A front with three dangerous self-tangencies and its marked chord diagram

Gromov's theorem of Sect. 1.2 implies that two fronts $C_{0}$ and $C_{1}$ with the same marked $n$-chord diagram are related by a homotopy $\left\{C_{t}\right\}_{t \in[0,1]}$ in which any front $C_{t}$ has $n$ dangerous self-tangencies except for a finite number of instants $t$ when $C_{t}$ gets $n+1$ dangerous self-tangencies (cf. [12]). Thus the symbol of an invariant of order $n$ defines a function on marked chord diagrams with $n$ chords. The main result of this section (Proposition 2) is a description of this function, denoted by $\left\langle D_{C}\right\rangle_{n}$, for the symbol of the coefficient $\langle C\rangle_{n}$ at $t^{n}$ of $\left.\langle C\rangle\right|_{A=e^{t}}$ (cf. Sect. 6.3 of [5]).

To formulate the statement we redraw Fig. 10 in terms of diagrams. Order chords $c_{1}, \ldots, c_{n}$ of an abstract marked $n$-chord diagram $D$ in an arbitrary way. Define two signed splittings of a chord $c_{i}$ as shown in Fig. 12. In each of the cases the chord is substituted by two oriented marked arcs. As it will become obvious from what follows, these splittings of $c_{i}$ correspond exactly to the similarly signed splittings of the self-tangency point $d_{i}$ in Fig. 10 if the chord represents the point in the marked diagram of a front.
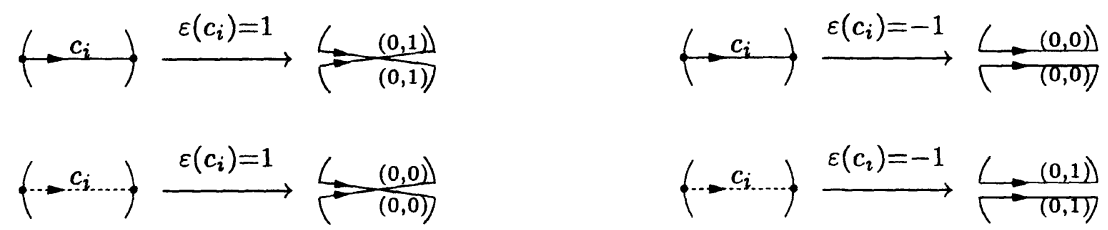

Fig. 12. Splittings of chords, their signs and markings

Let $D_{\varepsilon_{1}, \ldots, \varepsilon_{n}}$ be a splitting of the diagram $D$ with signs $\varepsilon\left(c_{i}\right)=\varepsilon_{i}$. We denote by $l\left(\varepsilon_{1}, \ldots, \varepsilon_{n}\right)$ the number of components of $D_{\varepsilon_{1}, \ldots, \varepsilon_{n}}: D_{\varepsilon_{1}, \ldots, \varepsilon_{n}}=\cup_{j}\left(D_{\varepsilon_{1}, \ldots, \varepsilon_{n}}\right)_{j}$. Each component consists of oriented marked arcs which are either arcs of circles of $D$ or the results of splittings of chords of $D$. For a component $\left(D_{\varepsilon_{1}, \ldots, \varepsilon_{n}}\right)_{j}$ we define 

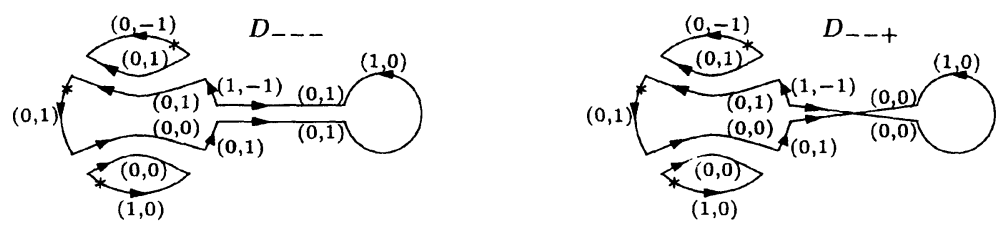

$$
\begin{array}{ll}
\imath_{1}=0-0=0 & i_{3}=1-0=1 \\
\mu_{1}=-1-1=-2 & \mu_{3}=0-0=0 \\
i_{2}=0+0+0+0+1-0+1+0=2 \\
\mu_{2}=1+0+1+1+0-1-1+1=2
\end{array}
$$

$$
\begin{array}{ll}
\imath_{1}=0-0=0 & i_{3}=1-0=1 \\
\mu_{1}=-1-1=-2 & \mu_{3}=0-0=0 \\
i_{2}=0+0+0+0-1-0+1+0=0 \\
\mu_{2}=1+0+1+0-0-0-1+1=2
\end{array}
$$

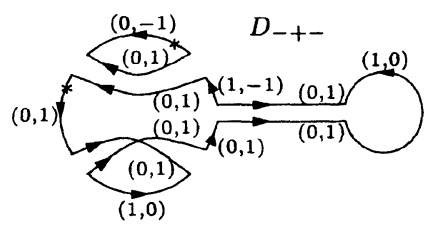

$$
\begin{aligned}
& i_{1}=0-0=0 \quad \mu_{1}=-1-1=-2 \\
& i_{2}=0+0-1+0+0+0+1-0+1+0=1 \\
& \mu_{2}=1+1-0+1+1+1+0-1-1+1=4
\end{aligned}
$$

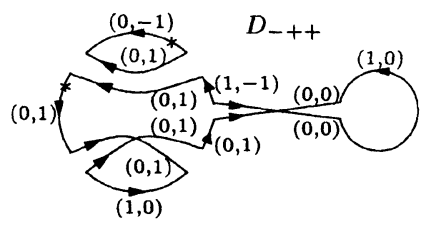

$$
\begin{aligned}
& i_{1}=0-0=0 \quad \mu_{1}=-1-1=-2 \\
& i_{2}=0+0-1+0+0+0-1-0+1+0=-1 \\
& \mu_{2}=1+1-0+1+1+0-0-0-1+1=4
\end{aligned}
$$

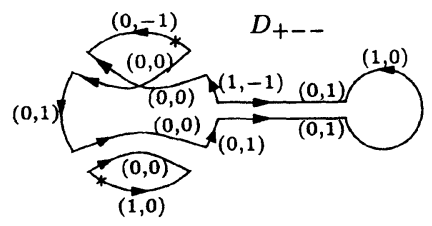

$$
\begin{aligned}
& i_{1}=0-0-1+0-1-0-0-0-0-0=-2 \\
& \mu_{1}=-1-0+1+1-0-1-1-0-1-0=-2 \\
& i_{2}=1-0=1 \quad \mu_{2}=0-0=0
\end{aligned}
$$

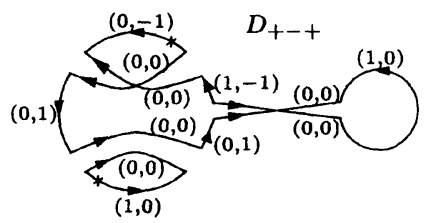

$$
\begin{aligned}
& i_{1}=0-0-1+0+1-0-0-0-0-0=0 \\
& \mu_{1}=-1-0+1+0+0-0-1-0-1-0=-2 \\
& i_{2}=1-0=1 \quad \mu_{2}=0-0=0
\end{aligned}
$$

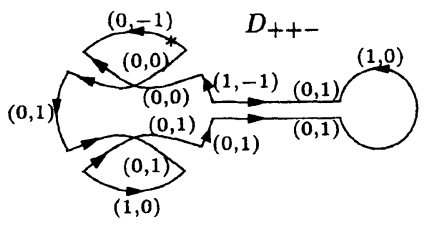

$$
\begin{aligned}
& i_{1}=0-0-1+0-1-0-0-0+1-0-0-0=-1 \\
& \mu_{1}=-1-0+1+1-0-1-1-1+0-1-1-0=-4
\end{aligned}
$$

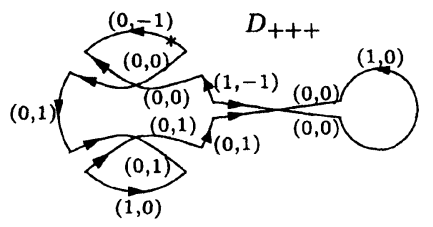

$$
\begin{aligned}
& i_{1}=0-0-1+0+1-0-0-0+1-0-0-0=1 \\
& \mu_{1}=-1-0+1+0+0-0-1-1+0-1-1-0=-4
\end{aligned}
$$

Fig. 13. Eight splittings of the marked chord diagram $D$ of Fig. 11. We assume the chords enumerated as in Fig. 11. Calculating the indices of a component we are walking along the component starting from the point $*$ in the direction of the arc containing $*$

two integers, index $i_{j}$ and Maslov index $\mu_{j}$, as follows (see Fig. 13). Let us walk along the component $\left(D_{\varepsilon_{1}, \ldots, \varepsilon_{n}}\right)_{j}$ and sum markings $(i, \mu)$ of the arcs we visit with appropriate signs. Walking along an arc oriented in (resp. opposite to) the direction of our journey we take its index $i$ and Maslov index $\mu$ with the sign plus (resp. minus). Of course, $i_{j}$ and $\mu_{j}$ change their signs for the trip in the opposite direction. But the statement below does not depend on these signs. 
Proposition 2. The value $\langle D\rangle_{n}$ of the $n^{\text {th }}$ coefficient of the Kauffman bracket on a marked chord diagram $D$ is given by the formula

$$
\langle D\rangle_{n}=-2^{n} \sum_{\varepsilon_{1}, \ldots, \varepsilon_{n}} 2^{l\left(\varepsilon_{1}, \cdots, \varepsilon_{n}\right)-1} \cdot \varepsilon_{1} \cdot \cdots \cdot \varepsilon_{n} \cdot \prod_{j=1}^{l\left(\varepsilon_{1}, \ldots, \varepsilon_{n}\right)}(-1)^{\mu_{j} / 2} T_{\left|i_{j}\right|}(h)
$$

where the sum is taken over all $2^{n}$ possible splittings of $D$, the product is taken over all components of a splitting, $T_{n}(\cos x)=\cos (n x)$ are the classical Tchebyshev polynomials.

Example. For the marked chord diagram $D$ of Fig. 11 we have the eight splittings shown in Fig. 13. Therefore

$$
\begin{aligned}
\langle D\rangle_{3}= & -8\left(-4\left(-T_{0}(h)\right)\left(-T_{2}(h)\right) T_{1}(h)+4\left(-T_{0}(h)\right)\left(-T_{0}(h)\right) T_{1}(h)\right. \\
& +2\left(-T_{0}(h)\right) T_{1}(h)-2\left(-T_{0}(h)\right) T_{1}(h)+2\left(-T_{2}(h)\right) T_{1}(h) \\
& \left.-2\left(-T_{0}(h)\right) T_{1}(h)-T_{1}(h)+T_{1}(h)\right) \\
= & -8 T_{1}(h)\left(-4 T_{0}(h)\left(T_{2}(h)-T_{0}(h)\right)-2\left(T_{2}(h)-T_{0}(h)\right)\right) \\
= & 16 T_{1}(h)\left(T_{2}(h)-T_{0}(h)\right)\left(2 T_{0}(h)-1\right) \\
= & 16 h\left(2 h^{2}-1-1\right)(2-1)=32 h\left(h^{2}-1\right),
\end{aligned}
$$

since $T_{0}(h)=1, T_{1}(h)=h, T_{2}(h)=2 h^{2}-1$.

Proof of Proposition 2. Let $C$ be a front with $n$ dangerous self-tangencies. The proof of Theorem 2 (see Sect. 3.1) provides an explicit formula for the value $\langle C\rangle_{n}$ of the $n^{\text {th }}$ coefficient of the Kauffman bracket on this front:

$$
\langle C\rangle_{n}=2^{n} \sum_{\varepsilon_{1}, \ldots, \varepsilon_{n}} \varepsilon_{1} \cdot \cdots \cdot \varepsilon_{n} \cdot\left\langle C_{\varepsilon_{1}, \ldots, \varepsilon_{n}}\right\rangle_{0}
$$

So for calculation of $\langle C\rangle_{n}=\left\langle D_{C}\right\rangle_{n}$ it is enough to know the zero order coefficients $\left\langle C_{\varepsilon_{1}, \ldots, \varepsilon_{n}}\right\rangle_{0}$. The lemma below gives an explicit formula for $\left\langle C_{\varepsilon_{1}, \ldots, \varepsilon_{n}}\right\rangle_{0}$ in terms of absolute values of indices and Maslov indices of components of the front $C_{\varepsilon_{1}, \ldots, \varepsilon_{n}}$. When the ordering of the chords in the diagram $D_{C}$ is induced by an ordering of dangerous self-tangencies of the front $C$, these absolute values are easily seen to be given by the above algorithm of counting the index information about the splitting $\left(D_{C}\right)_{\varepsilon_{1}, \ldots, \varepsilon_{n}}$. Thus Proposition 2 follows from

Lemma 5. Let $C=\cup_{j=1}^{l} C_{j}$ be a normal front with l components. Put $i_{j}=\operatorname{ind}\left(C_{j}\right)$ and $\mu=\sum_{j=1}^{l} \mu\left(C_{j}\right)$. Then

$$
\langle C\rangle_{0}=-2^{l-1}(-1)^{\mu / 2} \prod_{j=1}^{l} T_{\left|i_{j}\right|}(h),
$$

where $T_{n}(\cos x)=\cos (n x)$ are the classical Tchebyshev polynomials. 
Proof of Lemma 5. The second equality of Proposition 1 of Sect. 2.1 implies that the zero order coefficient of the Kauffman bracket is invariant under dangerous selftangencies as well. Due to Gromov's theorem (Sect. 1.2) $\langle C\rangle_{0}$ depends only on indices and Maslov indices of components of $C$. Therefore it is enough to calculate $\langle C\rangle_{0}$ on a collection of canonical curves. Property 4) of Theorem 1 implies

$$
\left\langle C_{1} \cdot C_{2}\right\rangle_{0}=-2\left\langle C_{1}\right\rangle_{0} \cdot\left\langle C_{2}\right\rangle_{0}
$$

So Lemma 5 follows from the calculation of the Kauffman bracket on the canonical curves from Sect. 2.4 .

Remark. Proposition 2 shows that the orientations of chords in a marked chord diagram do not matter for the value of the symbol of the coefficient. Indeed reorientation of a chord in a diagram $D$ can affect only the Maslov indices $\mu_{j}$ in the formula of the proposition. But for any splitting of the diagram the sum of the $\mu_{j}$ modulo 4 is not affected.

In fact the independence from orientations of chords is a general property of the symbol of any $J^{+}$-type invariant $f$ :

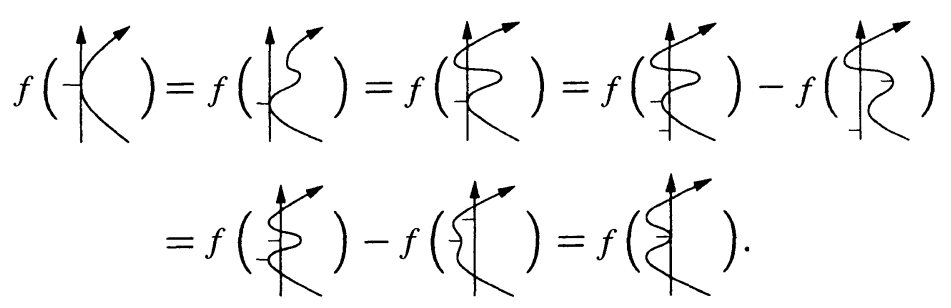

The second equality here is due to the fact that we are considering a symbol. The $3 \mathrm{rd}$ and 5 th ones are the definition. A similar chain of equalities is valid for an inverse dangerous self-tangency.

Thus the orientation of chords in our definition of the marked chord diagram of a front with dangerous self-tangencies should be omitted.

The obtained relation is not the only relation on the values of symbols on our marked chord diagrams. There are a lot of others, some of which are quite obvious. A complete diagrammatic description of symbols of finite order $J^{+}$-type invariants of one component plane fronts has been obtained by J.W. Hill [15]. It turns out that one needs to add one more marking, by the Maslov index of the whole front, to the marked chord diagrams used in [12] in the case of a regular plane curve.

3.3. The first coefficient. The proposition below means that the first coefficient $\langle C\rangle_{1}$ of the Kauffman bracket of a one component normal front $C$ carries the same information as the quantum Bennequin invariant $\beta_{q}\left(L_{C}\right)$ from Sect. 1.4. Setting $h=\left(q+q^{-1}\right) / 2$ brings $\langle C\rangle_{1}$ to the form whose essential part is $\beta_{q}\left(L_{C}\right)$. A reason for this substitution is that it makes the Tchebyshev polynomials $T_{n}(h)$ very simple: $T_{n}\left(\frac{q+q^{-1}}{2}\right)=\frac{q^{n}+q^{-n}}{2}=\left([n+1]_{q}-[n]_{q}\right) / 2$. Unfortunately the explicit formula relating $\langle\stackrel{2}{C}\rangle_{1}$ and $\beta_{q}\left(L_{C}\right)$ does not look very elegant. 
Proposition 3. Let $C$ be a one component normal front of index $i$ and Maslov index $\mu$. Then

$$
\left.\langle C\rangle_{1}\right|_{h=\left(q+q^{-1}\right) / 2}=(-1)^{\mu / 2}\left(\frac{1}{2}\left(q^{i}+q^{-i}\right) J^{+}(C)+2 \beta_{q}\left(L_{C}\right)\right)+R(i, \mu),
$$

where the quantum constant $R(i, \mu)$ depends only on the index $i$ and Maslov index $\mu$ of $C$ :

$$
\begin{aligned}
R(i, \mu)= & (-1)^{\mu / 2}\left([|i|+2]_{q}-\left(|i|+|\mu|+\frac{3}{2}\right)[|i|+1]_{q}\right. \\
& \left.-\left(|i|-\frac{|\mu|}{2}-\frac{3}{2}\right)[|i|]_{q}+\left(2|i|+\frac{|\mu|}{2}+2\right)[|i|-1]_{q}+[|i|-2]_{q}\right) .
\end{aligned}
$$

Proof of Proposition 3. We have to check two points. Firstly, the values of both sides of the identity on the canonical curves should coincide. Secondly, the jumps of both sides should be the same under a dangerous self-tangency perestroika.

The fact that the canonical curves satisfy the identity follows from the direct computations. We actually introduced the complicated term $R(i, \mu)$ as the difference between the values of the left-hand side and the remaining part of the right-hand side on the curve $K_{|i|+1,|\mu| / 2}$ with any orientation and coorientation (both sides of the identity do not change when we either reorient or recoorient a front). The evaluation of the "main" part of the right-hand side on the canonical curves is provided by the settings and computations of Sects. 1.3 and 1.4. The left-hand side of the identity is $\left.\frac{\partial}{\partial A}\langle C\rangle\right|_{A=1}$. Its evaluation on the canonical curves is based on rather elementary calculations (we omit them here) of similar derivatives of the deformations of the Tchebyshev polynomials of Sect. 2.3.

Now $R(i, \mu)$ does not change under any perestroika. So the jump of the righthand side of the identity under a dangerous self-tangency perestroika at a point $d$ of index $i_{d}$ is equal (see Sects. 1.3 and 1.4) to

$$
(-1)^{\mu / 2}\left(q^{i}+q^{-i}+2\left(q^{i_{d}}+q^{-i_{d}}\right)\right) \text {. }
$$

Let us calculate the jump of the left-hand side. According to Sect. 3.2 we associate one of the marked chord diagrams of Fig. 14 to a dangerous self-tangency point $d$ of index $i_{d}$.

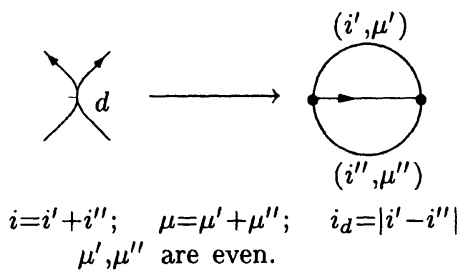

direct self-tangency

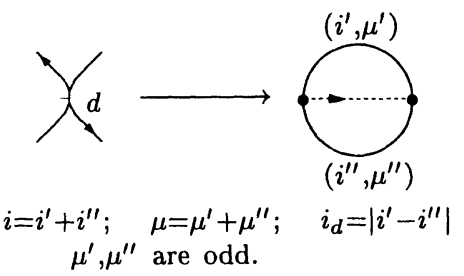

inverse self-tangency

Fig. 14. Marked chord diagrams of a self-tangency point $d$ of index $i_{d}$

The jump is the value of the symbol of $\langle C\rangle_{1}$ on the corresponding diagram. By Proposition 2 (Sect. 3.2) for calculation of the values we have to consider two splittings of each of the diagrams as it is shown in Fig. 15. 


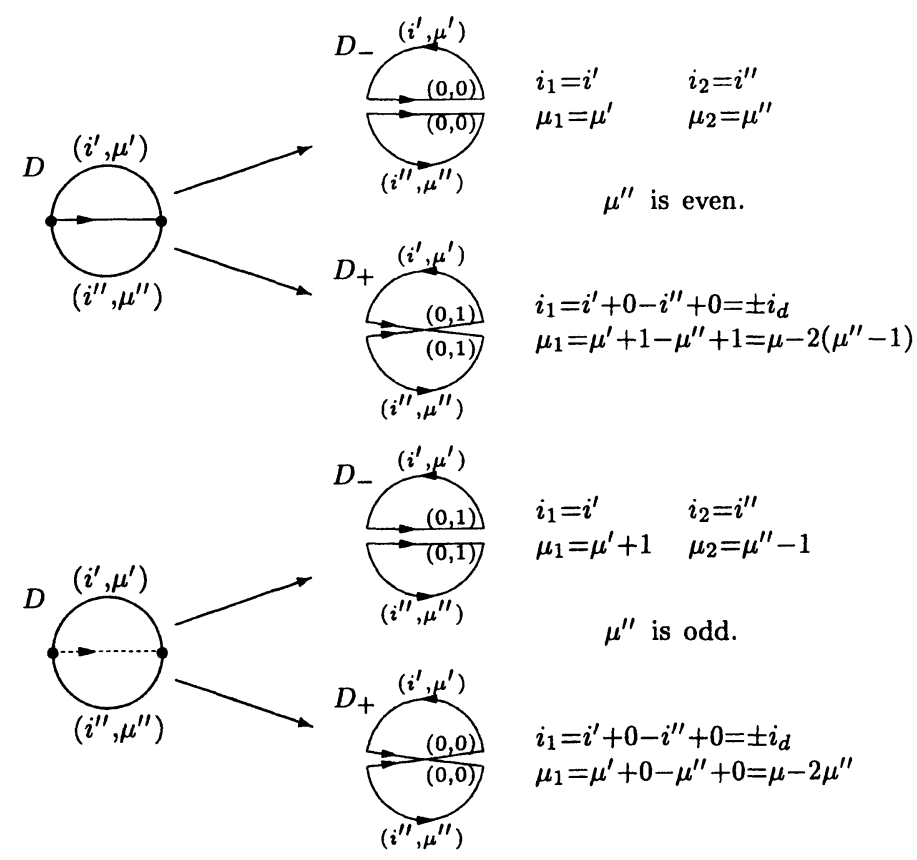

Fig. 15. Splittings of marked chord diagrams with one chord

Therefore the jumps are as follows.

For a direct self-tangency ( $\mu^{\prime \prime}$ is even):

$$
\begin{aligned}
& \left\langle-\langle\rangle_{1}-\langle\rangle-\langle\rangle_{1}\right. \\
& =-2\left(-2(-1)^{\mu^{\prime} / 2} T_{\left|i^{\prime}\right|}(h)(-1)^{\mu^{\prime \prime} / 2} T_{\left|i^{\prime \prime}\right|}(h)+(-1)^{\mu / 2-\left(\mu^{\prime \prime}-1\right)} T_{i_{d}}(h)\right) \\
& =2(-1)^{\mu / 2}\left(2 T_{\left|i^{\prime}\right|}(h) T_{\left|i^{\prime \prime}\right|}(h)+T_{i_{d}}(h)\right) \\
& =2(-1)^{\mu / 2}\left(2 \cdot \frac{q^{\prime}+q^{-i^{\prime}}}{2} \cdot \frac{q^{i^{\prime \prime}}+q^{-1^{\prime \prime}}}{2}+\frac{q^{i^{\prime} d}+q^{-t}}{2}\right) \\
& =(-1)^{\mu / 2}\left(q^{l}+q^{-i}+2\left(q^{i_{d}}+q^{-l_{d}}\right)\right) .
\end{aligned}
$$

For an inverse self-tangency ( $\mu^{\prime \prime}$ is odd):

$$
\begin{aligned}
\langle\rangle & \langle\rangle_{1}-\left\langle-\langle\rangle_{1}\right. \\
& =-2\left(-2(-1)^{\left(\mu^{\prime}+1\right) / 2} T_{\left|i^{\prime}\right|}(h)(-1)^{\left(\mu^{\prime \prime}-1\right) / 2} T_{\left|i^{\prime \prime}\right|}(h)+(-1)^{\mu / 2-\mu^{\prime \prime}} T_{i_{d}}(h)\right) \\
& =2(-1)^{\mu / 2}\left(2 T_{\left|i^{\prime}\right|}(h) T_{\left|i^{\prime \prime}\right|}(h)+T_{i_{d}}(h)\right) \\
& =(-1)^{\mu / 2}\left(q^{i}+q^{-i}+2\left(q^{i_{d}}+q^{-t_{d}}\right)\right) .
\end{aligned}
$$

Proposition 3 is proved. 


\section{Other Polynomials}

Similar to the definition of the Kauffman bracket of a plane front, the Legendrian lifting lowers other polynomial invariants of knots in a solid torus to normal fronts. Below we rewrite the HOMFLY and Kauffman polynomials of [21] in terms of plane curves and formulate a series of corresponding conjectures. The difficulty in proving these conjectures is that there is no obvious direction in application of the calculation rules, unlike the knot case where such a direction is to pass to an unknot by changes of crossings in a knot diagram.

4.1. HOMFLY polynomial. The HOMFLY polynomial of an oriented unframed link in a solid torus [21] is an element of $\mathbf{Z}\left[x^{ \pm 1}, y^{ \pm 1}, z_{ \pm 1}, z_{ \pm 2}, \ldots\right]$. The Legendrian translation of the definition of its obvious framed version (which takes values in the same polynomial ring and will be denoted by $P$ ) gives the following relations

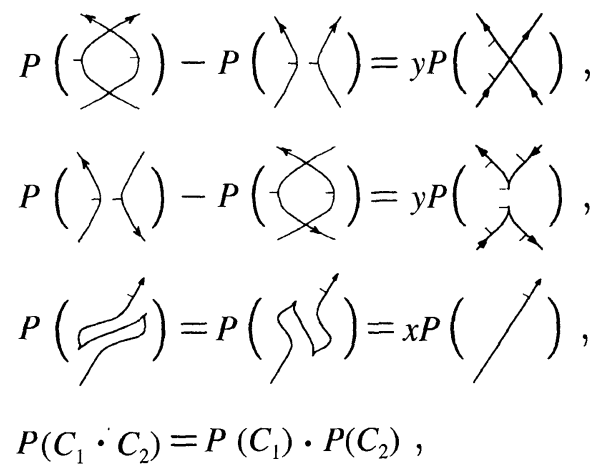

and initial data

$$
\begin{aligned}
& P(\longmapsto)=\frac{1-x^{2}}{y}, \\
& P(\text { r..m })=z_{l} \text {, for the curve of winding number } i \neq 0 .
\end{aligned}
$$

Relations of the first three lines are valid for the fragments with the reversed orientations as well; $C_{1} \cdot C_{2}$ is the disjoint union of the two non-empty fronts on different sides of a certain straight line.

Conjecture 1. There exists a unique $J^{+}$-type invariant

$$
P(C) \in \mathbf{Z}\left[x^{ \pm 1}, y^{ \pm 1}, z_{ \pm 1}, z_{ \pm 2}, \ldots\right]
$$

of an oriented normal front $C$ satisfying the above relations and initial data.

The way in which one uses relations of the third line in calculations of the polynomials in not very complicated cases (for example, for the curves of the tables from [3]) allows us to make

Conjecture 2. For any normal front $C$ the unique invariant $P(C)$ of Conjecture 1 is a genuine polynomial (not a Laurent one) in $x$. 
Conjecture 2 would imply a new estimate on the Bennequin number of a Legendrian knot in the standard contact solid torus.

4.2. Kauffman polynomial. The Kauffman polynomial $L$ of a framed non-oriented link in a solid torus [21] is an element of $\mathbf{Z}\left[x^{ \pm 1}, y^{ \pm 1}, z_{1}, z_{2}, \ldots\right]$. Lowering of the rules of its calculation to normal non-oriented plane fronts provides the relations

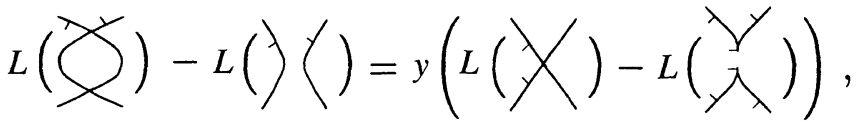

$$
\begin{aligned}
& L(S)=L(S S)=x L(/) \text {, } \\
& L\left(C_{1} \cdot C_{2}\right)=\left(\frac{1-x^{2}}{y}+x\right) \cdot L\left(C_{1}\right) \cdot L\left(C_{2}\right),
\end{aligned}
$$

along with the initial data

$$
\begin{aligned}
& L(\longrightarrow)=1, \\
& L(\text { m.m })=z_{t} \text { for the curve with } 2 i+2 \geqq 4 \text { cusps } .
\end{aligned}
$$

The fronts $C_{1}$ and $C_{2}$ here are similar to the ones in the relation for the HOMFLY polynomial.

Conjecture 3. There exists a unique $J^{+}$-type invariant $L(C) \in \mathbf{Z}\left[x^{ \pm 1}, y^{ \pm 1}, z_{1}, z_{2}, \ldots\right]$ of a non-oriented normal front $C$ satisfying the above relations and initial data.

Once again the numerical experiments dictate

Conjecture 4. For any normal front $C$ the unique invariant $L(C)$ of Conjecture 3 is a genuine polynomial (not a Laurent one) in $x$.

The latter would imply yet one more new estimate for the Bennequin invariant of a Legendrian knot.

4.3. The standard $\mathbf{R}^{3}$. Take a normal plane front with each component of Whitney index zero and with no vertical tangents. We will call such a front non-vertical.

Consider a non-vertical front as the graph of a multi-valued function on the horizontal axis. Taking the derivative as the third coordinate lifts it to a Legendrian link in $\mathbf{R}^{3}$ with the standard contact structure of the space of 1 -jets of functions on a line. Any generic Legendrian link in this standard $\mathbf{R}^{3}$ is the lift of a non-vertical front.

Thus we can lower the HOMFLY and Kauffman polynomials of framed knots in $\mathbf{R}^{3}$ to non-vertical fronts assuming them cooriented upwards. In comparison with the rules for arbitrary normal fronts, we have to omit the definitions of the variables $z_{i}$ and rotate all the fragments in the relations by 90 degrees clockwise.

Similar to Conjectures 1,3 and 4, the sufficiency of the obtained rules to calculate the polynomials of a non-vertical front is under question, along with the version of the Kauffman polynomial being a true polynomial in $x$. The corresponding version of Conjecture 2 for the HOMFLY polynomial of a Legendrian knot has been 
recently proved by D.Fuchs and S.Tabachnikov [10]. According to the result of [10], this implies the $\mathbf{R}^{3}$-version of Conjecture 4 modulo 2 .

Acknowledgements. This work was started when the authors were participating in the Semester on low-dimensional topology at Dipartimento di Matematica of Università di Pisa. The authors are thankful to R. Benedetti and M. Galbiati for the invitations. The first author thanks S. Duzhin, M. Polyak, S. Tabachnikov for stimulating discussions and Max-Planck-Institut für Mathematik in Bonn for warm hospitality.

\section{References}

1. Aicardi, F.: Topological invariants of knots and framed knots in the solid torus. C.R. Acad. Sci. Paris 321, Série I, 81-86 (1995)

2. Aicardi, F.: Topological invariants of legendrian curves. C.R. Acad. Sci. Paris 321, Série I, 199-204 (1995)

3. Arnold, V.I.: Topological invariants of plane curves and caustics. University Lecture Series 5. Providence, RI: AMS, 1994

4. Arnold, V.I.: Invariants and perestroikas of wave fronts on the plane. Trudy Mat. Inst. Steklova 209, 14-64 (in Russian) (1995)

5. Bar-Natan, D.: On the Vassiliev knot invariants. Topology 34, 423-472 (1995)

6. Bennequin, D.: Enterlacements et équation de Pfaff. Astérisque 107-108, 87-162 (1983)

7. Birman, J., Lin, X.-S.: Knot polynomials and Vassiliev invariants. Invent. Math. 111, 225-270 (1993)

8. Chmutov, S., Duzhin, S.: Explicit formulas for Arnold's generic curve invariants. In: ArnoldGelfand Mathematical Seminars. Geometry and Singularity Theory. Birkhäuser, 1996, to appear

9. Ferrand, E.: On the Bennequin invariant and the geometry of wave fronts. Preprint (1995)

10. Fuchs, D., Tabachnikov, S.: Invariants of Legendrian and transverse knots in the standard contact space. Topology, to appear

11. Goryunov, V.: Vassiliev invariants of knots in $\mathbf{R}^{3}$ and in a solid torus. Preprint 1-95, The University of Liverpool (1995)

12. Goryunov, V.: Vassiliev type invariants in Arnold's $J^{+}$-theory of plane curves without direct self-tangencies. Preprint 2-95, The University of Liverpool (1995)

13. Goryunov, V.: Local invariants of mappings of surfaces into three-space. In: Arnold-Gelfand Mathematical Seminars. Geometry and Singularity Theory. Birkhäuser, 1996, to appear

14. Gromov, M.: Partial differential relations. Berlin and New York: Springer-Verlag, 1986

15. Hill, J.W.: Vassiliev-type invariants in $J^{+}$-theory of planar fronts without dangerous selftangencies. Preprint, The University of Liverpool (1996)

16. Hoste, J., Przytycki, J.H.: An invariant of dichromatic links. Proc. AMS 105, 1003-1007 (1989)

17. Kauffman, L.: Knots and Physics. Singapore: World Scientific Press, 1991

18. Polyak, M.: Invariants of plane curves and Legendrian fronts via Gauss diagrams. Preprint, Bonn: Max-Planck-Institut für Mathematik (1994)

19. Polyak, M.: On the Bennequin invariant of Legendrian curves and its quantization. Preprint, Bonn: Max-Planck-Institut für Mathematik (1995)

20. Tabachnikov, S.: Computation of the Bennequin invariant of a Legendrian curve from the geometry of its front. Funk. Anal. i ego Pril. 22, no. 3, 89-90 (1988); English translation: Func. Anal. Appl. 22, 246-248 (1988)

21. Turaev, V.G.: The Conway and Kauffman modules of the solid torus with an appendix on the operator invariants of a tangle. LOMI preprint E-6-88, Leningrad (1988) 
